\title{
Bulk magnetic domain structures visualized by neutron dark-field imaging
}

\author{
C. Grünzweig, ${ }^{1, a)}$ C. David, ${ }^{1}$ O. Bunk,${ }^{1}$ M. Dierolf, ${ }^{1}$ G. Frei, ${ }^{1}$ G. Kühne,${ }^{1}$ R. Schäfer, ${ }^{2}$ \\ S. Pofahl, ${ }^{2}$ H. M. R. Rønnow, ${ }^{3}$ and F. Pfeiffer ${ }^{1,3, b)}$ \\ ${ }^{1}$ Paul Scherrer Institut, CH-5232 Villigen PSI, Switzerland \\ ${ }^{2}$ IFW Dresden, Institute for Metallic Materials, Helmholtzstrasse 20, D-01069 Dresden, Germany \\ ${ }^{3}$ Ecole Polytechnique Fédérale de Lausanne, CH-1015 Lausanne, Switzerland
}

(Received 9 July 2008; accepted 31 July 2008; published online 16 September 2008)

\begin{abstract}
We report on how a neutron grating interferometer can yield projection images of the internal domain structure in bulk ferromagnetic samples. The image contrast relies on the ultrasmall angle scattering of unpolarized neutrons at domain wall structures in the specimen. The results show the basic domains of (110)-oriented sheets in an FeSi test sample. The obtained domain structures could be correlated with surface sensitive magneto-optical Kerr effect micrographs. () 2008 American Institute of Physics. [DOI: 10.1063/1.2975848]
\end{abstract}

Weiss postulated in 1907 that magnetic samples are subdivided into elementary domains, each having an arbitrary magnetization direction. The observation of these domains was achieved on the surface by Bitter in 1931 with the help of an improved powder method. Today a variety of approaches exist for the observation of surface domains such as Kerr microscopy and magnetic force microscopy. Moreover, one can image domains in thin film samples provided they are transparent for electrons (Lorentz microscopy) or $\mathrm{x}$ rays (spectromicroscopy). ${ }^{1,2}$ The investigation of the internal domain structure in bulk metallic materials, however, still remains a significant challenge. The exceptional case is the Libovicky method. ${ }^{3,4}$ However, the method is only applicable for alloys with a composition of $\mathrm{Fe} 12.8$ at. \% Si. Moreover, this method is destructive as the specimen has to be cut to access the internal domains. The advantage of neutrons in this context is that they can easily penetrate centimeter thick metallic samples and interact through their spin directly with the local magnetization. ${ }^{5}$ This stimulated the development of other investigation techniques, ${ }^{6}$ which are, for example, based on single-crystal interferometry ${ }^{7-9}$ or on crystal analyzer-based topography. ${ }^{10,11}$ Unfortunately, these techniques suffer from the intrinsic limitations of crystal optics, which can tolerate only a very small beam divergence and a small energy spread. These circumstances lead to very inefficient setups and impair broader applications of such methods.

Here we report on an approach that overcomes these limitations and yields spatially resolved projection images of the internal domain structure in a bulk ferromagnetic sample with unprecedented quality. The approach is based on a grating interferometer setup implemented with polychromatic neutrons. In Fig. 1(a) the experimental setup is shown. It consists of a source grating (G0), a phase grating (G1), and an analyzer attenuation grating (G2). ${ }^{12}$ We have previously shown that such a setup can be used to obtain differential phase-contrast images ${ }^{13}$ and decoherence images (DCIs) that can be used to characterize magnetization processes in bulk in ferromagnetic samples. ${ }^{14}$

\footnotetext{
${ }^{a)}$ Electronic mail: christian.gruenzweig@psi.ch.

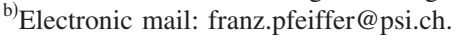

When one of the gratings is scanned along the transverse direction $x_{g}$ [see Fig. 1(b)], the intensity signal $I(m, n)$ in each pixel $(m, n)$ in the detector plane oscillates as a function of $x_{g}$ as depicted in Fig. 1(c). ${ }^{12,14,15}$ This intensity oscillation can be written as a Fourier series,

(a)

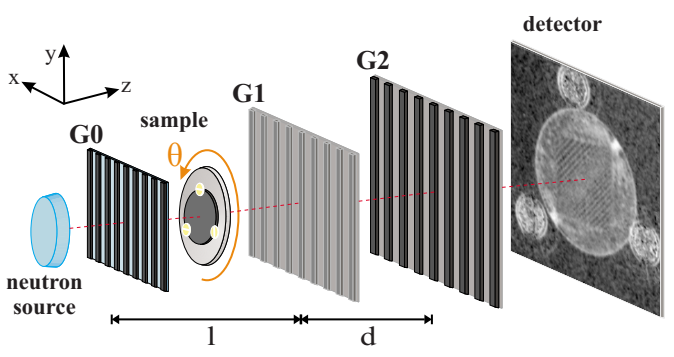

(b)

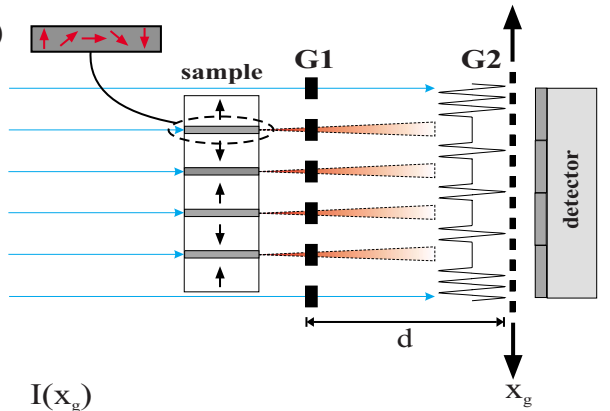

(c)

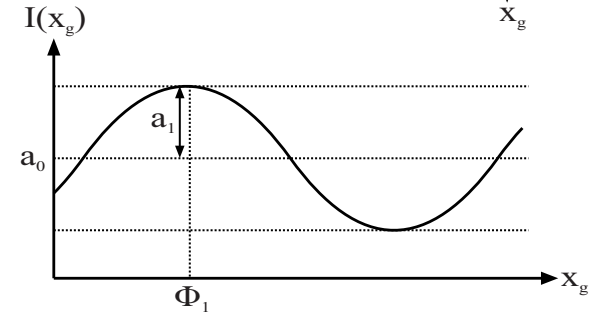

FIG. 1. (Color online) Neutron grating interferometer. (a) Setup showing the source grating (G0) $\left(p_{0}=1.08 \mathrm{~mm}\right)$, the phase grating (G1) $\left(p_{1}\right.$ $=7.97 \mu \mathrm{m})$ at a distance $l=5.23 \mathrm{~m}$, and the analyzer absorption grating (G2) $\left(p_{2}=4.00 \mu \mathrm{m}\right)$. (b) Through the Talbot effect a linear periodic fringe pattern is created behind G1 at a distance $d=19.4 \mathrm{~mm}$. Neutrons scattered at magnetic domain walls in the specimen locally degrade the interference pattern in the plane of G2. (c) Intensity modulation recorded in the detector pixel (without sample) when one of the gratings is scanned along $x_{g}$. 
(a)
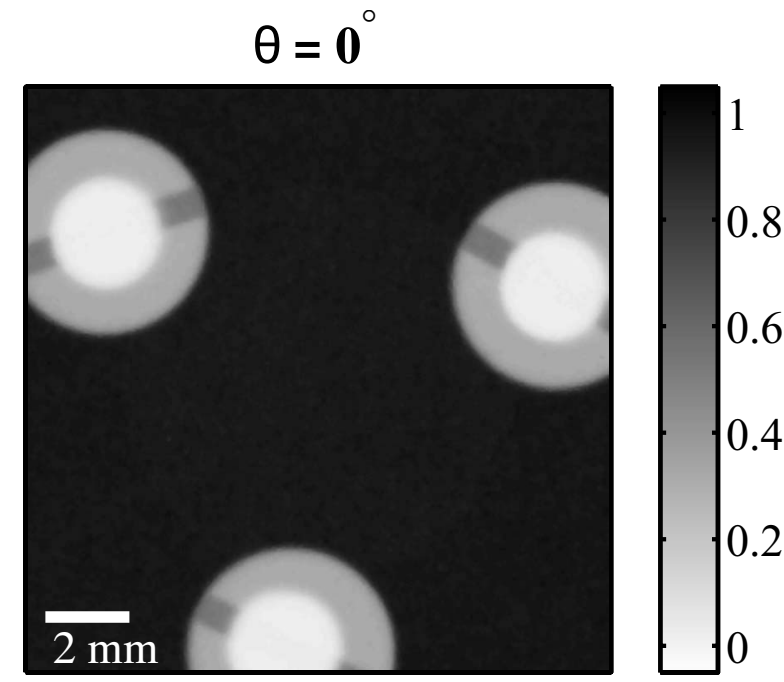

(c)
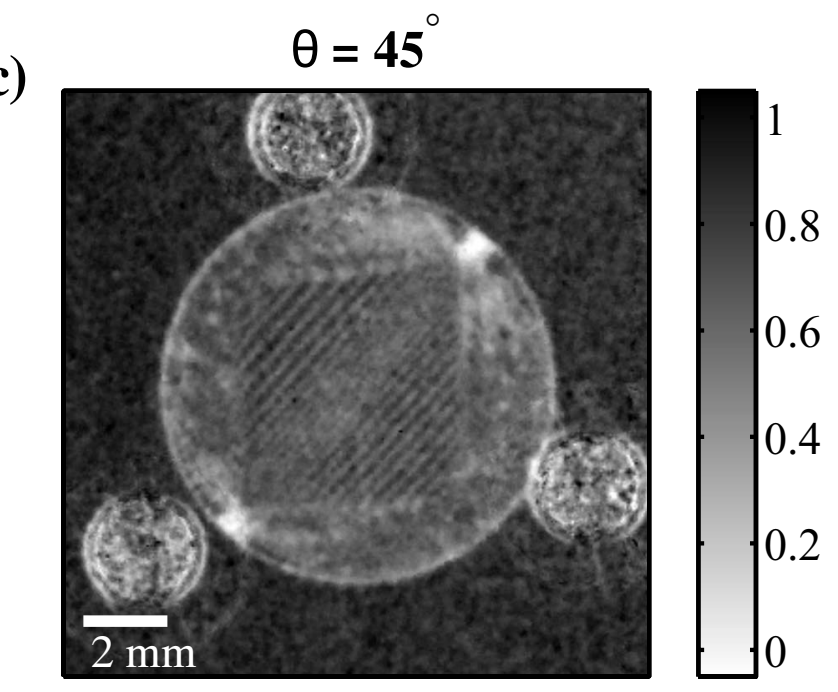
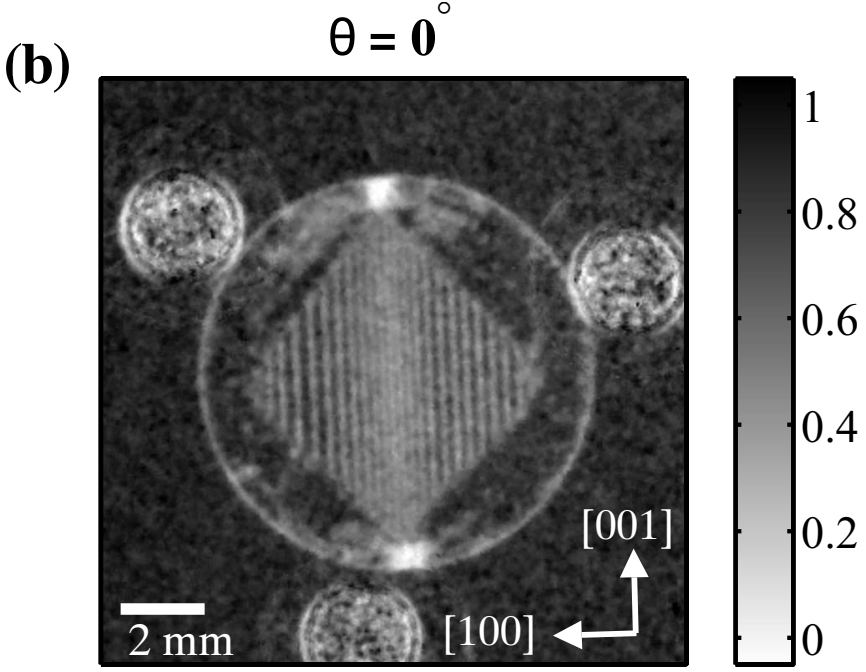

(d)

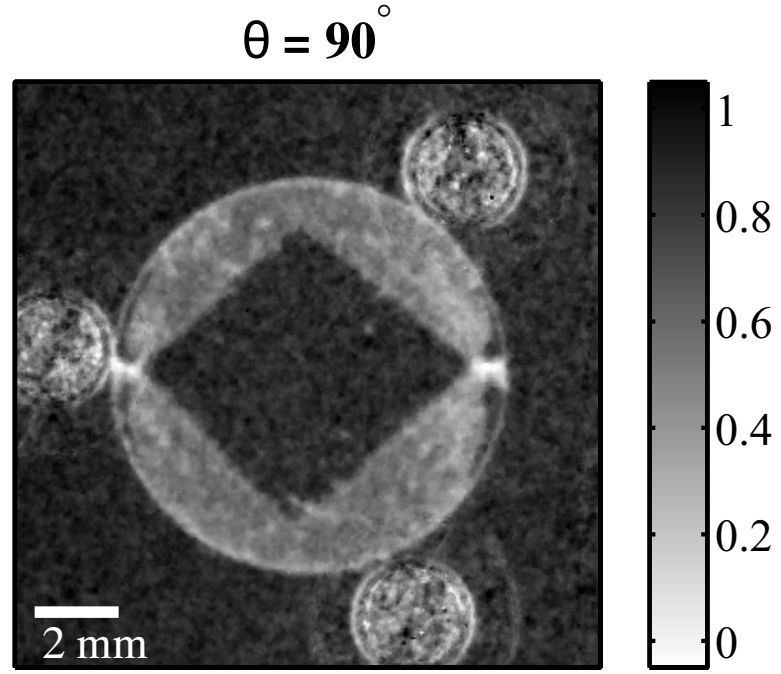

FIG. 2. Neutron results for the (110)-oriented FeSi single-crystal disk. (a) Conventional neutron transmission image. [(b)-(d)] DCIs showing a projection of the domain structure in the sample for several sample orientations $(\theta)$ [see Fig. 1(a)].

$$
\begin{aligned}
I\left(m, n, x_{g}\right)= & \sum_{i} a_{i}(m, n) \cos \left[i k x_{g}+\phi_{i}(m, n)\right] \approx a_{0}(m, n) \\
& +a_{1}(m, n) \cos \left[k x_{g}+\phi_{1}(m, n)\right],
\end{aligned}
$$

where $a_{i}$ are the amplitude coefficients, $\phi_{i}$ are the corresponding phase coefficients, $k \equiv 2 \pi / p_{2}$, and $p_{2}$ is the period of G2. The transmission image (identical to what would be measured with a conventional neutron radiography setup) is obtained by analyzing the zeroth Fourier component $a_{0}$ in each detector pixel of the specimen. It is given by $T(m, n)$ $=a_{0}^{s}(m, n) / a_{0}^{r}(m, n)$, where the superscripts $s$ and $r$ denote the values measured with the specimen in place and as a reference without, respectively. Spatially resolved information on the magnetic domain structure in the sample is obtained by analyzing the amplitude of the oscillation: $a_{1}(m, n) .{ }^{14}$ This analysis is based on the effect that the neutron beam undergoes multiple refractions at the domain boundaries (domain walls) in the sample, ${ }^{16}$ resulting in local degradation of the coherence of the neutron exit wave front behind the specimen. ${ }^{17}$ This local degradation decreases the ability of the neutrons to interfere with each other behind the phase grating (G1) and yields locally smaller values of the fringe visibility detected in the intensity oscillation $I\left(m, n, x_{g}\right)$ in certain detector pixels [see also Fig. 1(b)].
For a more quantitative description of this effect, we define the visibility of the intensity modulation in $I\left(m, n, x_{g}\right)$ by the ratio $V^{r}(m, n) \equiv\left(I_{\max }-I_{\min }\right) /\left(I_{\max }+I_{\min }\right)$ $=a_{1}^{r}(m, n) / a_{0}^{r}(m, n)$. Without the sample in place, this quantity contains information about the maximum contrast, which is produced by the grating interferometer. ${ }^{12}$ The relative decrease in this visibility due to the local coherence degradation caused by the specimen can then be quantified by defining the normalized visibility by $V(m, n) \equiv V^{s}(m, n) / V^{r}(m, n)$. Specimens that exhibit a strongly varying magnetic interaction potential show a significant decrease in the visibility with values of $V<1 .{ }^{14}$ Therefore we will refer to this image in the following as the neutron "DCI" or "dark-field image" (DFI) (Ref. 18) of the specimen.

The experiments were carried out at the Swiss Spallation Neutron Source using the beam port of the cold neutron imaging facility. ${ }^{19}$ A velocity selector was used to select neutrons with an average wavelength of $\lambda \simeq 4.1 \AA$ and a wavelength distribution (full width at half maximum) of $\Delta \lambda / \lambda$ $\simeq 16 \%$. A circular source with a diameter of $20 \mathrm{~mm}$ was used. Figure 2 displays results obtained for the test sample, a (110)-oriented iron silicon (FeSi) single-crystal disk. ${ }^{20,22}$ The conventional transmission image is shown in Fig. 2(a). Whereas only the strongly attenuating plastic screws used to 

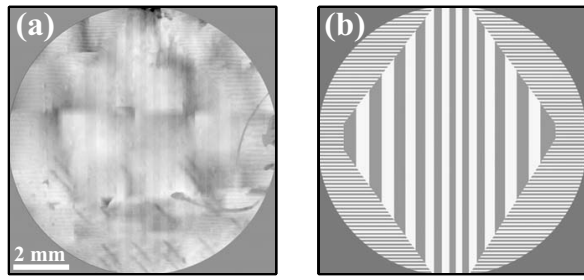

FIG. 3. MOKE image of the (110)-oriented FeSi single-crystal disk. (a) Kerr image of the surface domain structure. (b) Schematic of the obtained surface domain structure.

mount the disk are visible in the transmission image [Fig. 2(a)], the ferromagnetic disk is clearly visible in the DFI images [Figs. 2(b)-2(d)]. In Fig. 2(b) $\left(\theta=0^{\circ}\right)$ the sample was aligned with the [001] axis oriented parallel to the grating lines. Vertically oriented, elongated domain walls of several millimeters in length and several hundred microns in width are clearly visible in the DFI contrast. The good contrast produced by the domain walls in the DFI image arises from the strongly degraded neutron wave front and the corresponding small-angle scattering produced by the multiple refractions at the domain walls. The plastic screws, on the other hand, caused no significant contributions to the DFI since the plastic is a homogeneous material with essentially no density or magnetic fluctuations on the relevant length scale. The observed domain boundaries are associated with the basic domains of (110)-oriented FeSi sheets, which are magnetized along the surface-parallel easy [001] axis, separated by $180^{\circ}$ domain walls. ${ }^{1}$ We observe that the width of the domains, as measured by the distance between two domain walls, decreases (along the horizontal direction) with increasing distance from the center of the disk. Interestingly, the area where these domains are found forms a rhombohedron in the center of the specimen. Outside of this rhombohedral area, only very low contrast in the DFI is observed in Fig. 2(b). This changes dramatically when the sample is rotated by $\theta=45^{\circ}$ [Fig. 2(c)] or $\theta=90^{\circ}$ [Fig. 2(d)], respectively. The fact that the contrast outside of the rhombohedral area increases for increasing values of $\theta$ and reaches its maximum for $\theta=90^{\circ}$ indicates that the domains outside the rhombohedral area are oriented perpendicular to the ones inside the area, where an inverse behavior can be observed [see Figs. 2(b)-2(d)].

To interpret the observed DFI results that essentially are projection images of the internal bulk magnetic domain structure, we further recorded surface images of the same sample. For this we used a magneto-optical Kerr effect (MOKE) microscope. To obtain an equally large field of view, 40 single Kerr micrographs were combined into one representation. Figure 3(a) shows the result of the MOKE inspection, and Fig. 3(b) shows a schematic of the MOKE image. The rhombohedron with the inner domain structure is readily identified as well as the decreasing width of the domains with increasing distance from the center of the disk. Note that the DCI shows a frequency doubled image compared to the MOKE image since they show the domain walls. Moreover, the MOKE image shows fine horizontally aligned domain structures in the periphery of this rhombohedral area. The width of these domains is below the resolution of the neutron detector, and therefore they could not be individually resolved, e.g., in Fig. 2(d). These horizontal domains are the closure domains of internal basic domains that are magne- tized along the [100] and [010] directions, respectively. Such patterns are caused by compressive mechanical stress, which obviously dominates in the outer sample parts in a symmetric fashion. We note that the fine horizontal domain structure at the outer part of the rhombohedron does not contribute to the DFI shown in Fig. 2(b) $\left(\theta=0^{\circ}\right)$ since these domains cause scattering only in a direction parallel to the grating lines. In the same way, the domains in the inner part of the rhombohedron do not contribute to the DFI in Fig. 2(d) $\left(\theta=90^{\circ}\right)$. For the $\theta=45^{\circ}$ case, shown in Fig. 2(c), both the outer and the inner domain walls contribute to the DFI signal.

In conclusion, we have shown how a grating-based interferometer can provide spatially resolved images of the internal bulk magnetic domain structure in centimeter sized opaque ferromagnetic specimens. We observed projection images of domain walls formed by the basic domains of (110)-oriented FeSi sheets in an FeSi test sample. The results could successfully be correlated with surface sensitive MOKE images and help clarify the orientation dependence of the DFI results.

${ }^{1}$ A. Hubert and R. Schäfer, Magnetic Domains (Springer, Berlin, 1998).

${ }^{2} \mathrm{H}$. Hopster and H. P. Oepen, Magnetic Microscopy of Nanostructures (Springer, Berlin, 2005).

${ }^{3}$ S. Libovicky, Phys. Status Solidi A 12, 539 (1972).

${ }^{4}$ R. Schäfer and S. Schinnerling, J. Magn. Magn. Mater. 215-216, 140 (2000)

${ }^{5}$ O. Halpern and T. Holstein, Phys. Rev. 59, 940 (1941).

${ }^{6}$ O. Schärpf and H. Strothmann, Phys. Scr. T24, 58 (1988).

${ }^{7}$ M. Schlenker, W. Bauspiess, W. Gräff, U. Bonse, and H. Rauch, J. Magn. Magn. Mater. 15-18, 1507 (1980).

${ }^{8}$ S. Nakatani, H. Tomimitsu, T. Takahashi, and S. Kikuta, Jpn. J. Appl. Phys., Part 2 31, L1137 (1992).

${ }^{9}$ H. Rauch and S. A. Werner, Neutron Interferometry (Oxford University Press, Oxford, 2000).

${ }^{10}$ M. Schlenker and J. Baruchel, J. Appl. Phys. 49, 1996 (1978).

${ }^{11}$ J. Baruchel, Physica B (Amsterdam) 192, 79 (1993).

${ }^{12}$ C. Grünzweig, F. Pfeiffer, O. Bunk, T. Donath, G. Kühne, G. Frei, M. Dierolf, and C. David, Rev. Sci. Instrum. 79, 053703 (2008).

${ }^{13}$ F. Pfeiffer, C. Grünzweig, O. Bunk, G. Frei, E. Lehmann, and C. David, Phys. Rev. Lett. 96, 215505 (2006).

${ }^{14}$ C. Grünzweig, C. David, O. Bunk, M. Dierolf, G. Frei, G. Kühne, J. Kohlbrecher, R. Schäfer, P. Lejcek, H. M. R. Rønnow, and F. Pfeiffer, Phys. Rev. Lett. 101, 025504 (2008).

${ }^{15}$ F. Pfeiffer, M. Bech, O. Bunk, P. Kraft, E. F. Eikenberry, Ch. Brönnimann, C. Grünzweig, and C. David, Nat. Mater. 7, 134 (2008).

${ }^{16}$ O. Schärpf, J. Appl. Crystallogr. 11, 626 (1978).

${ }^{17}$ F. Pfeiffer, O. Bunk, C. Schulze-Briese, A. Diaz, T. Weitkamp, C. David, F. van der Veen, I. Vartanyants, and I. K. Robinson, Phys. Rev. Lett. 94, 164801 (2005).

${ }^{18}$ M. Strobl, C. Grünzweig, A. Hilger, I. Manke, N. Kardjilov, C. David, and F. Pfeiffer, "Neutron dark-field tomography," Phys. Rev. Lett. (to be published)

${ }^{19}$ G. Kühne, G. Frei, E. Lehmann, and P. Vontobel, Nucl. Instrum. Methods Phys. Res. A 542, 264 (2005).

${ }^{20}$ The (110)-oriented FeSi (Fe3 wt \% Si) disk had a thickness of $300 \mu \mathrm{m}$ and was cut from a Goss-oriented transformer steel with a diameter of 10 $\mathrm{mm}$. We note that surface polishing was only necessary to acquire the Kerr images; neutron DFI contrast can equally well be obtained on unpolished samples.

${ }^{21}$ C. Grünzweig, G. Frei, E. Lehmann, G. Kühne, and C. David, Rev. Sci. Instrum. 78, 053708 (2007).

${ }^{22}$ The images were recorded using a $100 \mu \mathrm{m}$ thick Li-6/ZnS converter and fluorescence screen with a 1:1 optical lens system and a cooled charge coupled device (Fingerlake Instrumentation) $(1024 \times 1024$ pixels, pixel size of $24 \times 24 \mu \mathrm{m}^{2}$ ). The effective spatial resolution was mainly determined by the intrinsic blurring in the scintillation screen to $100 \mu \mathrm{m}$ (Ref. 21). A typical exposure time for a single raw image was $30 \mathrm{~s}$; typically four or eight images were taken to yield one DCI. 